\title{
Planar Parametrization in Isogeometric Analysis
}

\author{
Gravesen, Jens; Evgrafov, Anton ; Nguyen, Dang-Manh; Nørtoft, Peter
}

Published in:

Mathematical Methods for Curves and Surfaces

Link to article, DOI:

10.1007/978-3-642-54382-1_11

Publication date:

2014

Document Version

Peer reviewed version

Link back to DTU Orbit

Citation (APA):

Gravesen, J., Evgrafov, A., Nguyen, D-M., \& Nørtoft, P. (2014). Planar Parametrization in Isogeometric Analysis. In Mathematical Methods for Curves and Surfaces: 8th International Conference, MMCS 2012, Oslo, Norway, June 28 - July 3, 2012, Revised Selected Papers Springer. Lecture Notes in Computer Science Vol. 8177 https://doi.org/10.1007/978-3-642-54382-1_11

\section{General rights}

Copyright and moral rights for the publications made accessible in the public portal are retained by the authors and/or other copyright owners and it is a condition of accessing publications that users recognise and abide by the legal requirements associated with these rights.

- Users may download and print one copy of any publication from the public portal for the purpose of private study or research.

- You may not further distribute the material or use it for any profit-making activity or commercial gain

- You may freely distribute the URL identifying the publication in the public portal 


\title{
Planar Parametrization in Isogeometric Analysis
}

\author{
Jens Gravesen ${ }^{1}$, Anton Evgrafov ${ }^{1}$, \\ Nguyen Dang Manh ${ }^{2}$, and Peter Nørtoft ${ }^{3 \star}$ \\ 1 DTU Compute, Technical University of Denmark, Denmark, \\ \{jgra, aaev\}@dtu.dk. \\ 2 Institute of Applied Geometry, Johannes Kepler University, Austria, \\ Manh.Dang_Nguyen@jku . at. \\ 3 Applied Mathematics, SINTEF ICT, Norway, \\ peter@noertoft.net.
}

\begin{abstract}
Before isogeometric analysis can be applied to solving a partial differential equation posed over some physical domain, one needs to construct a valid parametrization of the geometry. The accuracy of the analysis is affected by the quality of the parametrization. The challenge of computing and maintaining a valid geometry parametrization is particularly relevant in applications of isogemetric analysis to shape optimization, where the geometry varies from one optimization iteration to another. We propose a general framework for handling the geometry parametrization in isogeometric analysis and shape optimization. It utilizes an expensive non-linear method for constructing/updating a high quality reference parametrization, and an inexpensive linear method for maintaining the parametrization in the vicinity of the reference one. We describe several linear and non-linear parametrization methods, which are suitable for our framework. The non-linear methods we consider are based on solving a constrained optimization problem numerically, and are divided into two classes, geometry-oriented methods and analysisoriented methods. Their performance is illustrated through a few numerical examples.
\end{abstract}

Keywords: Isogeometric analysis, shape optimization, parametrization

\section{Introduction}

Isogeometric analysis is a modern computational method for solving partial differential equations (PDEs), which is based on a successful symbiosis between the variational techniques utilized in isoparametric finite element analysis with the geometric modelling tools from computer aided design [14,4]. A key ingredient of isogeometric analysis is the parametrization of the physical domain over which the PDE is posed, in many ways analogous to mesh generation in standard finite element analysis. Just as mesh quality affects the accuracy of a finite element approximation, the quality of the parametrization affects the accuracy of isogeometric analysis, see $[21,2,34,35]$.

* Presently at DTU Compute, Technical University of Denmark, Denmark. 
The question of computing and maintaining a valid geometry parametrization is particularly relevant in applications of isogemetric analysis to shape optimization problems, see e.g. $[11,22,23,26]$. Every time the geometry changes, that is, at every shape optimization iteration, one needs to update the parametrization in order to maintain the accuracy of the numerical approximation to the PDEs, governing the underlying physical model of the system. The algorithm for parametrization updates should therefore be (a) computationally inexpensive, as it is executed often; and (b) differentiable with respect to the variables determining the shape of the domain, which allows one to advantageously utilize gradient-based optimization algorithms thus reducing the total number of optimization iterations when compared with non-smooth or zero-order methods. One may again draw a parallel with the shape optimization based on the regular finite element analysis, which involves updating the mesh in between the shape optimization iterations.

The approach based on the discrete Coons patch [6] is a popular way of generating candidate parametrizations. This method is explicit and as a result it is very computationally inexpensive. Unfortunately, the resulting map needs not to be injective, and it is often necessary to invest further work in order to obtain even a valid, that is, a bijective parametrization. Even more work may be required to improve the quality of such a parametrization. Another approach to the same problem, which we have often utilized, is based on the spring model, cf. Section 3.1, in which the edges in the control net are modelled as elastic springs. In order to find a candidate parametrization one is required to solve a system of linear algebraic equations, thus rendering the method slightly more expensive than the discrete Coons patch. In our experience, however, the quality of the parametrizations obtained with this approach is slightly better.

If a good parametrization of a domain with a similar shape and patch layout is known, e.g., by using one of the methods in Section 4, one may employ one of the many methods developed for mesh generation $[9,10,29]$ in order to compute a domain parametrization. We will in particular consider mean value coordinates $[8,13]$, cf. Section 3.2. A new linear method of the same type is a quasi-conformal deformation method, cf. Section 3.3, which is inspired by conformal maps. Finally, any non-linear method may be linearized in the vicinity of a reference parametrization thereby resulting in a linear method.

We believe that no single linear method is capable of producing a high quality parametrization in all geometric configurations, and therefore we mainly investigate some non-linear methods. Many existing methods rely on the theory of harmonic functions on the physical domain. The method in [20] works on a triangulated volume and starts by constructing a parametrization of the boundary, i.e., the outer surface, using two harmonic functions with near orthogonal gradients. Then using harmonic functions in $3 \mathrm{D}$ the parametrization is propagated inwards to fill the entire volume. In [24] the inverse of the parametrization is constructed in a coordinate by coordinate fashion, using harmonic functions on the level set of the previously constructed coordinate functions. Finally the parametrization is defined as a tensor product spline approximation of the inverse 
map. The method in [25] demands that the inverse of the parametrization of a planar domain is a pair of harmonic functions and then proceeds to solving a uniquely solvable non-linear equation. This is mathematically equivalent to the last method in Section 4.1, where the Winslow functional is minimized. There is a unique minimizer whose inverse is the same pair of harmonic functions. The Winslow functional can also be interpreted as a condition number for the Jacobian and it is in that role that it is used in [12]. One may of course devise other methods based on the idea of finding extrema of geometric functionals, quantitatively assessing the quality of the parametrization, such as the area orthogonality functional and the Liao functional, cf. Section 4.1.

The final class of methods is based on estimating the approximation error and generating a parametrization that makes the estimate as small as possible. As test cases one can take problems with known analytical solutions and try to find the parametrization that minimizes the discrepancy between the exact and the numerical solutions, see $[21,34]$ for a $1 \mathrm{D}$ eigenvalue problem and a $2 \mathrm{D}$ heat conduction problem, respectively. In practice one of course does not know the exact solution so instead a suitable error estimator is utilized. In Section 4.2 we try three different error estimators, where the first one is similar to the one used in $[35]$.

The outline of the rest of this paper is as follows. In Section 2 we introduce the parametrization problem studied in this work, including the partial differential equation to be solved, namely Poisson's equation. In Section 3, we introduce three linear parametrization methods, and in Section 4, we describe a family of nonlinear, optimization-based parametrization methods based on two classes of quality measures, namely purely geometric and analysis-oriented measures. In Section 5, numerical results are presented, and in Section 6 we discuss extensions of the methods to shape optimization and to multiple patches. Finally, the current findings and some future challenges are summarized in Section 7.

\section{Parametrization for Partial Differential Equations}

In the following, we introduce the context in which the parametrization problem occurs, we formulate the parametrization problem, and we state a condition for the validity of a B-spline parametrization.

\subsection{The Setting: Poisson's Problem}

We consider a mixed boundary value problem for Poission's equation in two dimensions in a regular domain $\Omega \subset \mathbb{R}^{2}$ with piecewise-smooth boundary $\partial \Omega$. The boundary $\partial \Omega$ is represented as a closure of the union of two open disjoint subsets $\Gamma_{D} \neq \emptyset$ and $\Gamma_{N}$, on which we impose Dirichlet and Neumann boundary conditions. That is, we are interested in finding a function $u: \mathbb{R}^{2} \rightarrow \mathbb{R}$, such that 


$$
\begin{aligned}
-\Delta u & =f & & \text { in } \Omega, \\
u & =g & & \text { on } \Gamma_{D}, \\
\nabla u \cdot \mathbf{n} & =h & & \text { on } \Gamma_{N},
\end{aligned}
$$

where $f, g, h: \mathbb{R}^{2} \rightarrow \mathbb{R}$ are given, and $\mathbf{n}$ is the outwards facing boundary normal. In the weak form, the boundary value problem (1) reads: Find $u \in\left\{w \in H^{1}(\Omega)\right.$ : $\left.\left.w\right|_{\Gamma_{D}}=g\right\}$ such that

$$
\int_{\Omega} \nabla u \cdot \nabla v \mathrm{~d} A=\int_{\Omega} f v \mathrm{~d} A+\int_{\Gamma_{N}} h v \mathrm{~d} s .
$$

for all $v \in\left\{w \in H^{1}(\Omega):\left.w\right|_{\Gamma_{D}}=0\right\}$.

\subsection{The Challenge: Parametrize the Interior}

In order to utilize isogeometric analysis for solving the boundary value problem (1) numerically, a suitable geometry parametrization $\mathbf{X}$ of the domain $\Omega$ is required. Constructing such a parametrization is akin to the mesh generation step required for the standard finite element analysis. The parametrization impacts the accuracy of the numerical solution to the problem [34]. Expectedly, a higher quality parametrization allows for numerical solution with higher accuracy, all other things being equal.

Assuming that the domain $\Omega \subset \mathbb{R}^{2}$ may be parametrized using a single patch, the challenge in two dimensions reads: given a parametrization $\mathbf{Y}: \partial[0,1]^{2} \rightarrow \mathbb{R}^{2}$ of the boundary $\partial \Omega$, construct a parametrization of the interior $\mathbf{X}:[0,1]^{2} \rightarrow \mathbb{R}^{2}$, such that $\left.\mathbf{X}\right|_{\partial[0,1]^{2}}=\mathbf{Y}$.

In B-spline-based isogeometric analysis, the maps $\mathbf{Y}$ and $\mathbf{X}$ are splines, e.g.,

$$
\mathbf{X}(\xi, \eta)=\left(\begin{array}{l}
x(\xi, \eta) \\
y(\xi, \eta)
\end{array}\right)=\sum_{i, j} \mathbf{X}_{i, j} M_{i}(\xi) N_{j}(\eta)
$$

where $M_{i}$ and $N_{j}$ are B-splines defined by polynomial degrees and knots vectors and $\mathbf{X}_{i, j}$ are the control points. The equivalent challenge is now to specify the interior control points given the boundary control points $[34,2,11]$. This problem is sketched in Fig. 1.

\subsection{The Jacobian}

As we assume the boundary map $\mathbf{Y}=\left.\mathbf{X}\right|_{\partial[0,1]^{2}}$ is a parametrization, in particular a homeomorphism, the map $\mathbf{X}:[0,1]^{2} \rightarrow \Omega$ is a diffeomorphism if and only if the Jacobian

$$
\mathbf{J}=\left(\mathbf{X}_{\xi} \mathbf{X}_{\eta}\right)=\left(\begin{array}{ll}
\frac{\partial \mathbf{X}}{\partial \xi} & \frac{\partial \mathbf{X}}{\partial \eta}
\end{array}\right)=\left(\begin{array}{ll}
\frac{\partial x}{\partial \xi} & \frac{\partial x}{\partial \eta} \\
\frac{\partial y}{\partial \xi} & \frac{\partial y}{\partial \eta}
\end{array}\right)=\left(\begin{array}{ll}
x_{\xi} & x_{\eta} \\
y_{\xi} & y_{\eta}
\end{array}\right)
$$




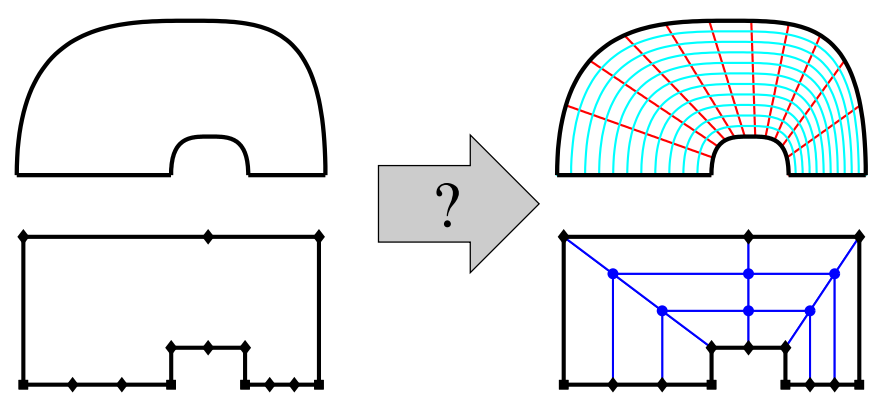

Fig. 1. Challenge: How do we go from a parametrization of the boundary of a domain to a parametrization that includes the interior of the domain?

is regular at every point. Therefore, in order to guarantee the validity of the parametrization, it is necessary that the determinant of the Jacobian does not vanish on $\Omega$. At the four corners of the parameter domain $[0,1]^{2}$, both partial derivatives of $\mathbf{X}$ are determined by the boundary parametrization $\mathbf{Y}$. As a consequence of this, there are domains which are impossible to parametrize. Indeed, consider the V-shaped domain in Fig. 2. If the boundary parametrization is reg-

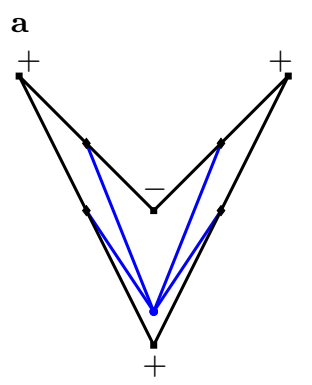

b

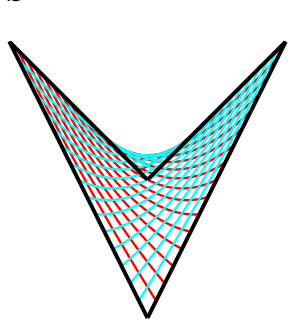

Fig. 2. An impossible domain. a: Control points and sign of the Jacobian determinant in the corners. b: The best quadratic parametrization when the edges are parametrized affinely.

ular, then the Jacobian has a positive determinant in the three convex corners, and a negative determinant in the concave corner (or vice versa if the orientation is reversed). So if the parametrization is $C^{1}$ on the closed parameter domain $[0,1]^{2}$, the determinant of the Jacobian attains both positive and negative values, and it is impossible to have a valid parametrization for this domain.

If we use B-splines to define the parametrization as in Equation (3), then the determinant can be written as

$$
\operatorname{det} \mathbf{J}=\sum_{i, j, k, \ell} \operatorname{det}\left(\begin{array}{ll}
x_{i, j} & x_{k, \ell} \\
y_{i, j} & y_{k, \ell}
\end{array}\right) M_{i}^{\prime}(\xi) N_{j}(\eta) M_{k}(\xi) N_{\ell}^{\prime}(\eta) .
$$


If $M$ and $N$ are B-splines of degree $p$ and $q$, respectively, this is clearly a piecewise polynomial map of degree $2 p-1$ in $\xi$ and of degree $2 q-1$ in $\eta$. As a result, it can be expressed in terms of B-splines $\widetilde{M}_{i}$ and $\widetilde{N}_{j}$ of degree $2 p-1$ and $2 q-1$, respectively, which are defined on the same knot vectors as $M_{i}$ and $N_{j}$ with multiplicities raised by $p$ and $q$ for interior knots and by $p-1$ and $q-1$ for the boundary knots. If rational NURBS are used, then we have a similar result, but the degree of $\widetilde{M}_{i}$ and $\widetilde{N}_{j}$ is now $3 p$ and $3 q$, respectively. In any case, we can write

$$
\operatorname{det} \mathbf{J}=\sum_{i, j} d_{i, j} \widetilde{M}_{i}(\xi) \widetilde{N}_{j}(\eta)
$$

As the B-splines $\widetilde{M}_{i}$ and $\widetilde{N}_{j}$ are non negative, we immediately obtain

Theorem 1. If the coefficients $d_{i, j}$ of the B-spline expansion (6) of the determinant of the Jacobian are positive then the parametrization is valid.

Observe that this is a sufficient condition and not a necessary one. However, if we perform knot insertion, then more and more coefficients will become positive. Indeed, if $\operatorname{det} \mathbf{J}>0$ on all of $[0,1]^{2}$, then $d_{i, j}>0$ for all $i, j$, after sufficiently many knot insertions. On the other hand, if the boundary parametrization has a zero derivative at some point, then the B-spline expansion (6) may have a negative coefficient no matter how many knot insertions we perform.

To demonstrate this, consider again the $\mathrm{V}$-shaped domain, but now assume that the boundary parametrization is quadratic and has a zero derivative at the concave corner $P_{1}$, see Fig. 3 . That is, the two edges meeting at $P_{1}$ are
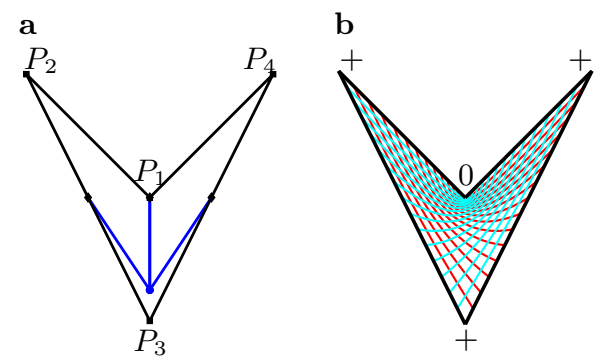

c

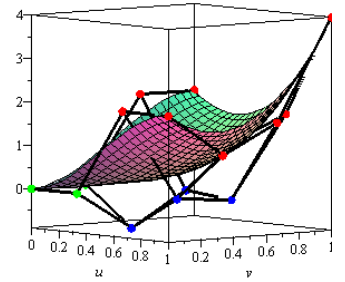

Fig. 3. The V-shaped domain with a singular boundary parametrization. a: Three control points are placed at the concave corner. b: The parametrization and the sign of the Jacobian determinant at the four corners. c: The Jacobian determinant.

parametrized as

$$
\left(1-\xi^{2}\right) P_{1}+\xi^{2} P_{2} \quad \text { and } \quad\left(1-\eta^{2}\right) P_{1}+\eta^{2} P_{4}
$$

respectively. By letting the single inner control point be $\frac{1}{4} P_{1}+\frac{3}{4} P_{3}$ we obtain a valid parametrization in the form of a bi-quadratic tensor product Bézier patch. 
We may assume that $P_{1}=\mathbf{0}$ and then

$$
\begin{aligned}
X(\xi, \eta) & =P_{2} B_{2}^{2}(\xi) B_{0}^{2}(\eta)+\frac{3}{4} P_{3} B_{1}^{2}(\xi) B_{1}^{2}(\eta)+P_{4} B_{0}^{2}(\xi) B_{2}^{2}(\eta) \\
& +\frac{P_{2}+P_{3}}{2} B_{2}^{2}(\xi) B_{1}^{2}(\eta)+\frac{P_{3}+P_{4}}{2} B_{1}^{2}(\xi) B_{2}^{2}(\eta)+P_{3} B_{2}^{2}(\xi) B_{2}^{2}(\eta) .
\end{aligned}
$$

The determinant of the Jacobian is a bi-cubic tensor product Bézier patch

$$
\operatorname{det} \mathbf{J}=\sum_{i, j=0}^{3} d_{i, j} B_{i}^{3}(\xi) B_{j}^{3}(\eta)
$$

We see that $d_{0,0}=d_{1,0}=d_{0,1}=0$, and $d_{1,1}=\operatorname{det}\left(P_{2}, P_{4}\right)<0$ but it is not hard to see that $\operatorname{det} \mathbf{J}>0$ on $] 0,1[2$, see Fig. 3 . This is still the case after any refinement of the knot vectors.

The fact that a change of the boundary parametrization of the V-shaped domain can make a parametrization of the interior possible was also noted in $[32]$.

\section{Linear Parametrization Methods}

In this section, we present three linear methods for computing geometry parametrizations. The first of these, the spring model, operates without the need for any information apart from the boundary parametrization; this method may therefore be utilized for generating initial parametrizations for other linear or non-linear methods. The last two, the mean value coordinates and the quasiconformal methods, rely on the knowledge of a reference parametrization of the interior. One may of course generate more linear methods by linearizing nonlinear ones around reference parametrizations, as discussed in Section 3.4.

\subsection{The Spring Model}

This method mimics a mechanical model, in which all edges in the control mesh are replaced with linear elastic springs. The mechanical equilibrium, which arises when the positions of the boundary control points are given, defines the position of the inner control points within this model. In this configuration, all inner control points are the averages of their four neighbours. That is, we have a set of simple linear equations:

$$
4 \mathbf{X}_{i, j}=\mathbf{X}_{i+1, j}+\mathbf{X}_{i-1, j}+\mathbf{X}_{i, j+1}+\mathbf{X}_{i, j-1}
$$

which is easily solved. By assigning different "spring constants" to different edges one obtains variations of the method. 


\subsection{Mean Value Coordinates}

In recent years there has been a lot of work on parametrization of polygonal meshes $[9,10,29]$. If we use the control point formulation of our spline parametrization problem, some of these methods can be applied to our problem. A popular and appealing method is based on the mean value coordinates $[8,13]$. Here, points in the plane are given as a particular affine combination of the vertices of a closed polygon. The closed polygon is in our case the boundary of the control net.

Suppose we are given a reference parametrization, with inner control points $\widehat{\mathbf{X}}_{k, \ell}$, and a set of boundary control points $\widehat{\mathbf{X}}_{i}, i=1, \ldots, n$, arranged in a counter clockwise fashion. Any point $\mathbf{x} \in \mathbb{R}^{2}$ can now be written as an affine combination of the boundary control points:

$$
\mathbf{x}=\sum_{i=1}^{n} \lambda_{i}(\mathbf{x}) \widehat{\mathbf{X}}_{i}, \quad \text { where } \quad \lambda_{i}(\mathbf{x})=\frac{w_{i}(\mathbf{x})}{\sum_{i=1}^{n} w_{i}(\mathbf{x})}
$$

The weights $w_{i}(\mathbf{x})$ are defined by

$$
\begin{aligned}
w_{i}(\mathbf{x})= & 2 \frac{\tan \left(\alpha_{i-1} / 2\right)+\tan \left(\alpha_{i} / 2\right)}{\left\|\widehat{\mathbf{v}}_{i}\right\|}=\frac{2}{\left\|\widehat{\mathbf{v}}_{i}\right\|}\left(\frac{\sin \alpha_{i-1}}{1+\cos \alpha_{i-1}}+\frac{\sin \alpha_{i}}{1+\cos \alpha_{i}}\right) \\
& =\frac{2}{\left\|\widehat{\mathbf{v}}_{i}\right\|}\left(\frac{\left[\widehat{\mathbf{v}}_{i-1}, \widehat{\mathbf{v}}_{i}\right]}{\left\|\widehat{\mathbf{v}}_{i-1}\right\|\left\|\widehat{\mathbf{v}}_{i}\right\|+\left\langle\widehat{\mathbf{v}}_{i-1}, \widehat{\mathbf{v}}_{i}\right\rangle}+\frac{\left[\widehat{\mathbf{v}}_{i}, \widehat{\mathbf{v}}_{i+1}\right]}{\left\|\widehat{\mathbf{v}}_{i}\right\|\left\|\widehat{\mathbf{v}}_{i+1}\right\|+\left\langle\widehat{\mathbf{v}}_{i}, \widehat{\mathbf{v}}_{i+1}\right\rangle}\right)
\end{aligned}
$$

where

$$
\langle\widehat{\mathbf{v}}, \widehat{\mathbf{w}}\rangle=v_{1} w_{1}+v_{2} w_{2} \quad \text { and } \quad[\widehat{\mathbf{v}}, \widehat{\mathbf{w}}]=v_{1} w_{2}-v_{2} w_{1}
$$

are the inner product and the determinant of a pair of vectors $\widehat{\mathbf{v}}$ and $\widehat{\mathbf{w}}$, respectively. The angles and vectors are defined in Fig. 4a. If we have a parametrization

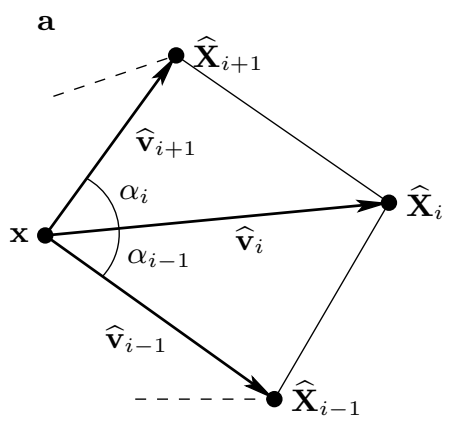

b

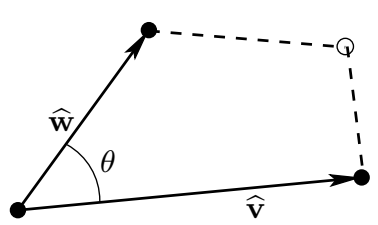

Fig. 4. a: Ingredients of mean value coordinates. b: Ingredients of the quasi conformal deformation.

of the boundary of another domain with new boundary control points $\mathbf{X}_{i}$, then 
we simply define the new inner control points as

$$
\mathbf{X}_{k, \ell}=\sum_{i=1}^{n} \lambda_{i}\left(\widehat{\mathbf{X}}_{k, \ell}\right) \mathbf{X}_{i}
$$

i.e., we use the same normalized weights as in the reference control net.

\subsection{Quasi Conformal Deformation}

Once again we assume we have a reference parametrization $\widehat{\mathbf{X}}$. The idea is that we would like any other parametrization to have a control net that locally looks like a conformal deformation of the reference control net.

Consider a quadrilateral and two neighbouring edges in the reference control net, cf. Fig. 4b. We think of these edges as vectors $\widehat{\mathbf{v}}$ and $\widehat{\mathbf{w}}$ emanating from their common vertex. The method is based on a simple geometric identity $\|\widehat{\mathbf{v}}\| \widehat{\mathbf{w}}=$ $\|\widehat{\mathbf{w}}\| \mathbf{R}(\theta) \widehat{\mathbf{v}}$, where $\mathbf{R}(\theta)$ is a rotation through the angle $\theta$, that is:

$$
\|\widehat{\mathbf{v}}\| \widehat{\mathbf{w}}=\|\widehat{\mathbf{w}}\| \mathbf{R}(\theta) \widehat{\mathbf{v}}=\frac{1}{\|\widehat{\mathbf{v}}\|}\left(\begin{array}{rr}
\langle\widehat{\mathbf{v}}, \widehat{\mathbf{w}}\rangle & -[\widehat{\mathbf{v}}, \widehat{\mathbf{w}}] \\
{[\widehat{\mathbf{v}}, \widehat{\mathbf{w}}]} & \langle\widehat{\mathbf{v}}, \widehat{\mathbf{w}}\rangle
\end{array}\right) \widehat{\mathbf{v}}
$$

If $\mathbf{v}$ and $\mathbf{w}$ are the corresponding edges in the new control net, then we can require that

$$
\|\widehat{\mathbf{v}}\| \mathbf{w}=\frac{1}{\|\widehat{\mathbf{v}}\|}\left(\begin{array}{lr}
\langle\widehat{\mathbf{v}}, \widehat{\mathbf{w}}\rangle & -[\widehat{\mathbf{v}}, \widehat{\mathbf{w}}] \\
{[\widehat{\mathbf{v}}, \widehat{\mathbf{w}}]} & \langle\widehat{\mathbf{v}}, \widehat{\mathbf{w}}\rangle
\end{array}\right) \mathbf{v}
$$

for each such pair of edges. For each inner control point we have four linear algebraic equations of the type (16), and for every boundary control point, apart from the corners, we have two equations. This results in $4(M N-M-N)$ equations in $(M-2)(N-2)$ unknown inner control points. The resulting overdetermined system is then solved in the least squares sense.

One could also look after a conformal deformation of the reference parametrization by replacing the vectors $\widehat{v}$ and $\widehat{w}$ with the partial derivatives $\widehat{\mathbf{X}}_{\xi}$ and $\widehat{\mathbf{X}}_{\eta}$. That is, the new parametrization $\mathbf{X}$ should satisfy the equation

$$
\left\|\widehat{\mathbf{X}}_{\xi}\right\| \mathbf{X}_{\eta}=\frac{1}{\left\|\widehat{\mathbf{X}}_{\xi}\right\|}\left(\begin{array}{cc}
\left\langle\widehat{\mathbf{X}}_{\xi}, \widehat{\mathbf{X}}_{\eta}\right\rangle & -\left[\widehat{\mathbf{X}}_{\xi}, \widehat{\mathbf{X}}_{\eta}\right] \\
{\left[\widehat{\mathbf{X}}_{\xi}, \widehat{\mathbf{X}}_{\eta}\right]} & \left\langle\widehat{\mathbf{X}}_{\xi}, \widehat{\mathbf{X}}_{\eta}\right\rangle
\end{array}\right) \mathbf{X}_{\xi}, \quad \text { in all of }[0,1]^{2}
$$

Similarly to the previous case, this family of equations could be solved in the least square sense.

\subsection{Linearized methods}

In the following section we will introduce several non-linear methods that work by minimizing a certain quality measure $c$, and by a linearization of these, we may obtain new linear methods. One way of formalizing this is by considering a second order Taylor expansion of the quality measure in the vicinity of a reference 
parametrization $\widehat{\mathbf{X}}$. If we let $\mathbf{X}_{1}$ denote the known control points, typically the boundary control points, and let $\mathbf{X}_{2}$ denote unknown control points, typically the inner control points, then we can write

$$
\begin{aligned}
c(\mathbf{X}) \approx & c(\widehat{\mathbf{X}})+\left(\mathbf{G}_{1}(\widehat{\mathbf{X}}) \mathbf{G}_{2}(\widehat{\mathbf{X}})\right)\left(\begin{array}{l}
\mathbf{X}_{1}-\widehat{\mathbf{X}}_{1} \\
\mathbf{X}_{2}-\widehat{\mathbf{X}}_{2}
\end{array}\right) \\
& +\frac{1}{2}\left(\mathbf{X}_{1}^{T}-\widehat{\mathbf{X}}_{1}^{T} \mathbf{X}_{2}^{T}-\widehat{\mathbf{X}}_{2}^{T}\right)\left(\begin{array}{l}
\mathbf{H}_{11}(\widehat{\mathbf{X}}) \mathbf{H}_{12}(\widehat{\mathbf{X}}) \\
\mathbf{H}_{21}(\widehat{\mathbf{X}}) \mathbf{H}_{22}(\widehat{\mathbf{X}})
\end{array}\right)\left(\begin{array}{l}
\mathbf{X}_{1}-\widehat{\mathbf{X}}_{1} \\
\mathbf{X}_{2}-\widehat{\mathbf{X}}_{2}
\end{array}\right),
\end{aligned}
$$

where $\mathbf{G}_{i}$ and $\mathbf{H}_{i j}$ gives the gradient and the Hessian of $c$ with respect to the control points of the parametrization. Assuming that the Hessian is positive definite, the right hand side is minimized when

$$
\mathbf{H}_{22}(\widehat{\mathbf{X}}) \mathbf{X}_{2}=\mathbf{H}_{22}(\widehat{\mathbf{X}}) \widehat{\mathbf{X}}_{2}-2 \mathbf{H}_{21}(\widehat{\mathbf{X}})\left(\mathbf{X}_{1}-\widehat{\mathbf{X}}_{1}\right)-\mathbf{G}_{2}(\widehat{\mathbf{X}}),
$$

which is a linear equation in the unknown control points $\mathbf{X}_{2}$.

\section{Nonlinear Parametrization Methods}

We proceed to presenting a family of nonlinear parametrization methods based on optimization, following the approach taken in, e.g. [34,35]. Thus, the interior parametrization is constructed by numerically maximizing quantitative measures of the parametrization quality. We divide these measure into two groups: the geometry-oriented and the analysis-oriented. Throughout, we assume that we are given a regular parametrization of the boundary with positive determinant of the Jacobian in the corners.

In order to have a valid parametrization, the Jacobian needs to have a nonvanishing determinant everywhere. Owing to our assumption about the sign in the corners, we will demand that the determinant is positive everywhere inside the domain, and we can then formulate the following max min problem

$$
\begin{array}{lr}
\underset{\mathbf{X}}{\operatorname{maximize}} & Z, \\
\text { such that } & \operatorname{det} \mathbf{J} \geq Z, \quad \text { in }[0,1]^{2}, \\
\text { where } & \left.\mathbf{X}\right|_{\partial[0,1]^{2}}=\mathbf{Y},
\end{array}
$$

In practice, we replace the condition (20b) with

$$
d_{i, j} \geq Z, \quad \text { for all } i, j,
$$

where $d_{i, j}$ are the coefficients of the determinant of the Jacobian, cf. (6). In case an optimization algorithm terminates with a configuration, for which we have $Z>0$, the resulting parametrization is necessarily valid. However, its quality does not have to be very high, cf. Fig. 5. Despite this drawback, the approach provides a simple way of generating valid initial parametrizations for other methods, which require such initialization. 

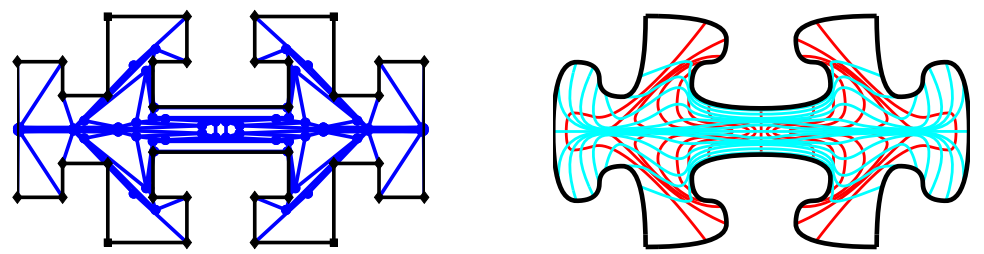

Fig. 5. Maximizing the smallest coefficient in the B-spline expansion of $\operatorname{det} \mathbf{J}$.

\subsection{Geometric Measures}

The first class of quality measures are geometric in nature, and thereby depend only on the parametrization itself. The methods in this class amount to solving an optimization problem, which can be formulated as

$$
\begin{array}{ll}
\underset{\mathbf{X}}{\operatorname{minimize}} & c(\mathbf{X}), \\
\text { such that } & \operatorname{det} \mathbf{J} \geq \delta Z, \quad \text { in }[0,1]^{2}, \\
\text { where } & \left.\mathbf{X}\right|_{\partial[0,1]^{2}}=\mathbf{Y},
\end{array}
$$

In the lower bound (22b) for $\operatorname{det} \mathbf{J}$, the number $\delta \in[0,1]$ is an algorithmic parameter and the number $Z$ is the result of the optimization (20). We have often successfully used $\delta=0$.

When defining geometric quality measures for a parametrization, the Jacobian $\mathbf{J}$ and the first fundamental form $g$ are important quantities:

$$
g=\mathbf{J}^{T} \mathbf{J}=\left[\begin{array}{cc}
x_{\xi}^{2}+y_{\xi}^{2} & x_{\xi} x_{\eta}+y_{\xi} y_{\eta} \\
x_{\xi} x_{\eta}+y_{\xi} y_{\eta} & x_{\eta}^{2}+y_{\eta}^{2}
\end{array}\right]
$$

With these in mind, we proceed to define the area-orthogonality, the Liao, and the Winslow functionals, which are all well-known quantities for mesh generations, see e.g. $[17,7]$.

The Area-Orthogonality Functional The area-orthogonality measure $m_{\mathrm{AO}}$ is defined as the product of the diagonal entries of the metric tensor $g,[7,15]$

$$
m_{\mathrm{AO}}=g_{11} g_{22}=\left(x_{\xi}^{2}+y_{\xi}^{2}\right)\left(x_{\eta}^{2}+y_{\eta}^{2}\right) .
$$

Based on this, we may define the area-orthogonality functional $c_{\mathrm{AO}}$ as the integral of the area-orthogonality measure $m_{\mathrm{AO}}$ over the parameter domain:

$$
c_{\mathrm{AO}}=\int_{0}^{1} \int_{0}^{1} m_{\mathrm{AO}} \mathrm{d} \xi \mathrm{d} \eta .
$$


The Liao Functional The Liao measure $m_{L}$ is defined as the Frobenius norm of the metric tensor $g$, i.e., the sum of the square of its entries $[18,17,7,15]$ :

$$
m_{\mathrm{L}}=g_{11}^{2}+g_{22}^{2}+2 g_{12}^{2}=\left(x_{\xi}^{2}+y_{\xi}^{2}\right)^{2}+\left(x_{\eta}^{2}+y_{\eta}^{2}\right)^{2}+2\left(x_{\xi} x_{\eta}+y_{\xi} y_{\eta}\right)^{2} .
$$

As above, we may define the Liao functional as

$$
c_{\mathrm{L}}=\int_{0}^{1} \int_{0}^{1} m_{\mathrm{L}} \mathrm{d} \xi \mathrm{d} \eta
$$

The Winslow Functional In this approach, the goal is to construct a parametrization as conformal as possible $[33,11,22]$.

The parametrization $\mathbf{X}$ is conformal if and only if the Jacobian $\mathbf{J}$ is the product of a scaling and a rotation, or, equivalently, if the first fundamental form $g$ is diagonal with identical diagonal elements. If we let $\lambda_{1}$ and $\lambda_{2}$ denote the eigenvalues of $g$, we need $\lambda_{1}=\lambda_{2}$ to have conformality. We easily find that

$$
\frac{\left(\sqrt{\lambda_{1}}-\sqrt{\lambda_{2}}\right)^{2}}{\sqrt{\lambda_{1} \lambda_{2}}}=\frac{\lambda_{1}+\lambda_{2}-2 \sqrt{\lambda_{1} \lambda_{2}}}{\sqrt{\lambda_{1} \lambda_{2}}}=\frac{\lambda_{1}+\lambda_{2}}{\sqrt{\lambda_{1} \lambda_{2}}}-2 .
$$

From this, we may define the Winslow measure $m_{W}$ :

$$
m_{W}=\frac{\lambda_{1}+\lambda_{2}}{\sqrt{\lambda_{1} \lambda_{2}}}=\frac{\operatorname{tr}(g)}{\sqrt{\operatorname{det}(g)}}=\frac{x_{\xi}^{2}+x_{\eta}^{2}+y_{\xi}^{2}+y_{\eta}^{2}}{x_{\xi} y_{\eta}-y_{\xi} x_{\eta}},
$$

where $\sqrt{\operatorname{det}(g)}=\operatorname{det}(\mathbf{J})$. As such, $m_{W}$ is a pointwise measure of conformality. Using the Winslow function $m_{W}$ we define the Winslow functional as:

$$
c_{W}=\int_{0}^{1} \int_{0}^{1} m_{W} \mathrm{~d} \xi \mathrm{d} \eta
$$

and use this as a global measure of conformality.

The Winslow functional has particularly nice mathematical properties. Indeed, if we switch the integration in (29) from the parameter domain $[0,1]^{2}$ to the physical domain $\Omega$, then we obtain

$$
c_{W}=\int_{\Omega}\left(\left(\frac{\partial \xi}{\partial x}\right)^{2}+\left(\frac{\partial \xi}{\partial y}\right)^{2}+\left(\frac{\partial \eta}{\partial x}\right)^{2}+\left(\frac{\partial \eta}{\partial y}\right)^{2}\right) \mathrm{d} A .
$$

This is the well known Dirichlet energy, and the unique minimizer is a pair of harmonic functions $\Omega \rightarrow[0,1]^{2}$ whose restriction to the boundary is the inverse $\mathbf{Y}^{-1}$ of the given boundary parametrization $\mathbf{Y}: \partial[0,1]^{2} \rightarrow \partial \Omega$. As the target $[0,1]^{2}$ is convex, the Radó-Kneser-Choquet theorem $[1,5,16,28]$ ensures that this pair of harmonic functions is a diffeomorphism on the interior. This means that our optimization problem (22), with the cost function (29), also has a unique minimum which is a diffeomorphism whose inverse is a pair of harmonic functions. This is not in conflict with the impossible domain shown in Fig. 2: the diffeomorphism is defined on the interior, and the maps may be non-differentiable at the boundary. In Fig. 6 we show the parametrization ensured by the theorem. Notice that the $y$ coordinate is not differentiable in the concave corner, so the Jacobian is not defined in that corner. 

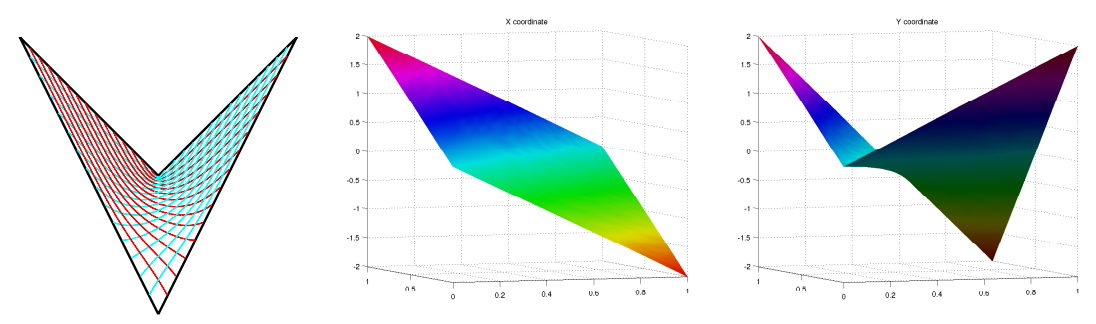

Fig. 6. Parametrization of the V-shape and the graphs of the $x$ and $y$ coordinates.

\subsection{Analysis-Oriented Measures}

In the other class of non-linear variational methods for constructing parametrizations, we put analysis-oriented methods. Here, the explicit goal is to construct as accurate analysis of a given partial differential equation as possible. This accuracy needs to be estimated, which can be done by comparing the solutions from several analyses or by evaluating the residual. In any case, when using methods in this class we aim at analysis-aware parametrizations $[34,2]$. The quality measure for these methods depends not only on the parametrization, but also on the solution to the PDE at hand (the Poisson problem (1) in our case). The resulting optimization problems can be formulated as follows:

$$
\begin{aligned}
& \underset{\mathbf{X}}{\operatorname{minimize}} \quad c(\mathbf{X}, u), \\
& \text { such that } \quad \operatorname{det} \mathbf{J} \geq \delta Z, \quad \text { in }[0,1]^{2}, \\
& \text { where }\left.\quad \mathbf{X}\right|_{\partial[0,1]^{2}}=\mathbf{Y} \text {, } \\
& -\Delta u=f, \quad \text { in } \Omega, \\
& u=g, \quad \text { on } \Gamma_{D}, \\
& \nabla u \cdot \mathbf{n}=h, \quad \text { on } \Gamma_{N} .
\end{aligned}
$$

As before, in the lower bound (31b) for $\operatorname{det} \mathbf{J}$, the number $\delta \in[0,1]$ is an algorithmic parameter and the number $Z$ is the result of the optimization (20). It goes without saying that if the Poisson problem is replaced by another problem, only the equations (31d)-(31f) are changed.

Strong Residual Norm. In this approach, we use the residual of the problem we are trying to solve as an error estimator. Hence, from Equation (31d) we set

$$
m_{\mathrm{SR}}=(\Delta u+f)^{2} .
$$

We emphasize that at least a quadratic B-spline approximation of the field $u$ must be employed. The exact expression for $m_{\mathrm{SR}}$ depends of course on the problem considered. As a result, we obtain the quality measure

$$
c_{\mathrm{SR}}=\int_{\Omega} m_{\mathrm{SR}} \mathrm{d} A .
$$


We could also consider the Neumann boundary condition (31f), which is only weakly satisfied, and add a term like $\alpha \int_{\Gamma_{N}}(\nabla u \cdot \mathbf{n}-h)^{2} \mathrm{~d} s$ to the cost function, where $\alpha$ is some weight factor.

Weak Residual Norm. Here, we again consider the residual, but instead of integrating it over the entire domain to get a global error estimator, we now project it onto a suitable space of test functions to obtain a set of local error estimators.

When the variational form of the PDE (2) is considered over a given space $\mathcal{S}_{1}$, the residual will belong to the orthogonal complement of this space owing to Galerkin's orthogonality. Therefore, we project the residual onto a larger space $\mathcal{S}_{2} \supsetneqq \mathcal{S}_{1}$ to obtain a meaningful, non-zero error estimator:

$$
m_{\mathrm{WR}, k}=\int_{\Omega} \nabla u \cdot \nabla R_{k} \mathrm{~d} A-\int_{\Gamma_{N}} g R_{k} \mathrm{~d} s-\int_{\Omega} f R_{k} \mathrm{~d} A .
$$

Here, the functions $R_{k}$ are the basis functions for $\mathcal{S}_{2}$ stemming from tensor product B-splines on the parameter domain $[0,1]^{2}$. There are many possibilities in choosing $\mathcal{S}_{2}$. One obvious choice is by halving all knot segments ( $h$-refinement), and another is degree elevation ( $p$-refinement). As the integration is performed knot segment by knot segment the latter yields cheaper integration, so this is the one we have tested. Again, the exact expression for $m_{\mathrm{WR}}$ depends on the problem considered.

In this method, we consider the quality measure

$$
c_{\mathrm{WR}}=\sum_{k} m_{\mathrm{WR}, k}^{2}
$$

Of course, we could also introduce weights $\alpha_{k}$ on $m_{\mathrm{WR}, k}$, e.g. the area of the support of the basis function $R_{k}$.

Enrichment Error Norm. As in the previous subsection we consider two different spline spaces $\mathcal{S}_{1} \varsubsetneqq \mathcal{S}_{2}$, but now we seek two approximate solutions $u_{1} \in \mathcal{S}_{1}$ and $u_{2} \in \mathcal{S}_{2}$ and regard their difference as an error estimator:

$$
u_{1}-u_{2}=\sum_{k} m_{\mathrm{EE}, k} R_{k}
$$

where the $R_{k}$ as above is the basis for $\mathcal{S}_{2}$. Therefore, the quality measure is

$$
c_{\mathrm{EE}}=\sum_{k} m_{\mathrm{EE}, k}^{2}
$$

and again, we could introduce weights $\alpha_{k}$ on $m_{\mathrm{EE}, k}$. Note that we have to solve the equation twice in this approach, so it is a rather expensive method. 


\section{$5 \quad$ Numerical Examples}

In this section, we study two numerical examples of the parametrization problem outlined in Section 2, and we compare the resulting parametrizations based on the nonlinear methods described in Section 4. The methods are implemented in MATLAB ${ }^{\circledR}[19]$ and Octave [27]. The optimization is done using IPOPT, a non-linear optimization package based on an interior point method [30]. In both examples, the geometries are represented by quadratic splines, while the scalar field $u$ is approximated using cubic splines. The equations are discretized using a Galerkin method as described in [4] and the knots are in all cases uniformly spaced. The weak residual and enrichment error methods are based on a degree elevation of the analysis spline by one, i.e., the spline spaces $\mathcal{S}_{1}$ and $\mathcal{S}_{2}$ consists of cubic and quartic $C^{2}$ splines, respectively.

\subsection{Poisson's Equation on a Wedge-Shaped Domain}

We consider the parametrization problem for a boundary value problem (BVP) with a known analytical solution. The example is taken from [34]. The domain under consideration is $\Omega=\left\{(x, y) \mid-1 \leq y \leq x^{2}, 0 \leq x \leq 1\right\}$, and we impose homogeneous boundary conditions $u=0$ on the entire boundary $\partial \Omega$, as depicted in Fig. 7a. The field $u^{*}=\sin \left(\pi\left(y-x^{2}\right)\right) \sin (\pi x) \sin (\pi y)$ obviously fulfills the
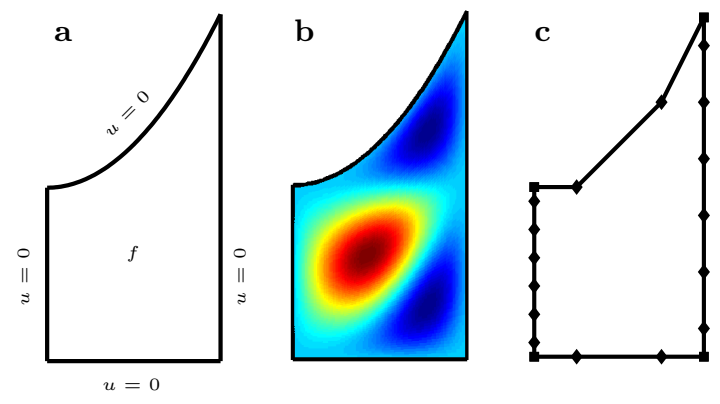

Fig. 7. Wedge-shaped domain. a: Domain and boundary conditions. b: Analytical solution of the boundary value problem. c: Boundary control points.

boundary conditions, and therefore is the unique solution to the BVP corresponding to $f=-\Delta u^{*}$. This solution is shown in Fig. $7 \mathrm{~b}$, and the control points of the boundary are depicted in Fig. $7 \mathrm{c}$.

We solve the parametrization problem for this BVP by optimizing the location of the 12 interior control points, yielding a total of 24 design variables for the optimization. We initialize all methods using the spring model in Section 3.1. Fig. 8 depicts, for each of the six parametrization methods, the optimal control net, the corresponding parametrization, and the numerical error, computed as the difference $\left|u_{h}-u^{*}\right|$ between the computed solutions $u_{h}$ and the analytical solution $u^{*}$. The depicted error is based on a discretization of the 
16
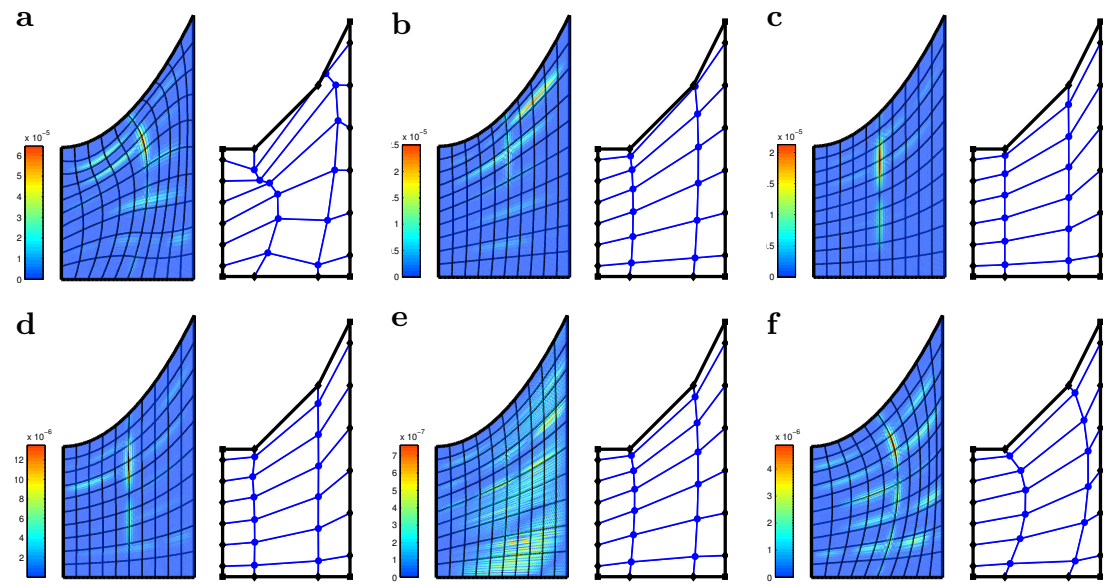

e
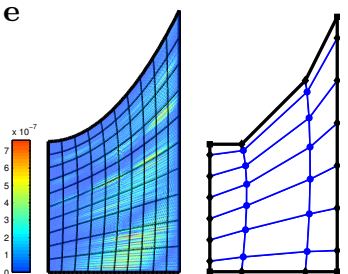

f
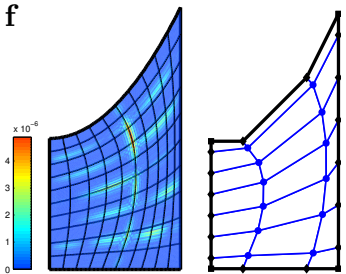

Fig. 8. Wedge-shaped domain: Isoparametric lines and numerical error (left) and control net (right) for the parametrization based on area-orthogonality (a), Liao (b), Winslow (c), strong residual (d), weak residual (e), and enrichment error (f).

state variable $u$ with $\sim 10^{4}$ degrees-of-freedom, while the optimization for the analysis-oriented methods are performed on a coarser discretization of $u$ with $\sim 10^{3}$ degrees-of-freedom. We note that the optimal control net and the corresponding parametrizations are quite similar for the Liao, the Winslow, the strong residual, and the weak residual methods, whereas the area-orthogonality, and the enriched error methods differ somewhat. This is also clearly reflected in the error, which is found to vary by several orders of magnitude between the methods.

An interesting question is, how well these parametrizations reproduce the analytical solution when we refine the analysis. The answer to this is shown in Fig. 9. The figure depicts the global numerical error $\epsilon$ as a function of the number of basis functions used to approximate the solution to the PDE for each of the six methods. As global numerical error, we use the $L_{2}$-norm of the local numerical error: $\epsilon^{2}=\int_{\Omega}\left|u_{h}-u^{*}\right|^{2} \mathrm{~d} A$. Note that for each method, the parametrization is kept fixed during these experiments. For not too coarse discretizations, we see that the error varies by several orders of magnitude between the methods, clearly emphasizing the importance of the way the domain is parametrized. The smallest error is found for the weak residual method, while the highest error is found for the area-orthogonality method. Additionally, for this example the error for the weak residual method converges faster than for the other methods, which have practically identical convergence orders.

We conclude this example by emphasizing that the computational expenses vary significantly between the two classes of methods. The geometrically based methods (area-orthogonality, Liao, and Winslow) converged within $\sim 30$ optimization iterations, whereas the analysis-oriented methods (strong residual, weak residual, and enrichment error) converged after $\sim 300$ iterations. Even 


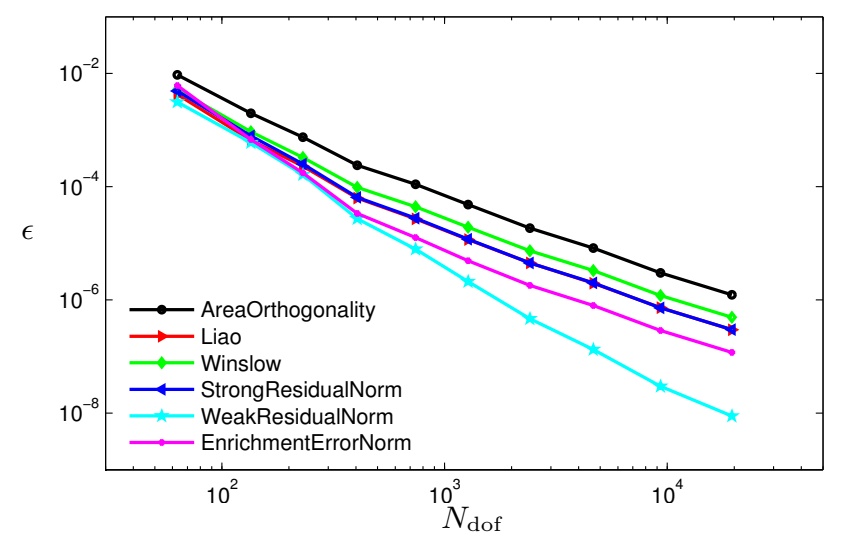

Fig. 9. Wedge-shaped domain: error as a function of number of degrees-of-freedom for the different parametrization methods.

more importantly, the analysis-oriented methods require solving the PDE in each optimization step, unlike the geometrical methods.

\subsection{Poisson's Equation on a Jigsaw puzzle}

We consider the Poisson problem (1) posed over the jigsaw puzzle piece shown in Fig. 10a. We use the field

$$
u_{G}^{*}=\sum_{i=1}^{2} \exp \left(-\frac{\left(x-\tilde{x}_{i}\right)^{2}}{a_{i}^{2}}-\frac{\left(y-\tilde{y}_{i}\right)^{2}}{b_{i}^{2}}\right)
$$

as boundary condition on $\partial \Omega$ with given parameters $\tilde{\mathbf{x}}, \tilde{\mathbf{y}}, \mathbf{a}, \mathbf{b} \in \mathbb{R}^{2}$, and with $f=-\Delta u_{G}^{*}, u_{G}^{*}$ is the unique solution to the BVP. The field is depicted in Fig. 10b. The boundary conditions are enforced strongly through the least square fit of the traces in the trial space to the field (38). The boundary control points are shown in Fig. 10c.

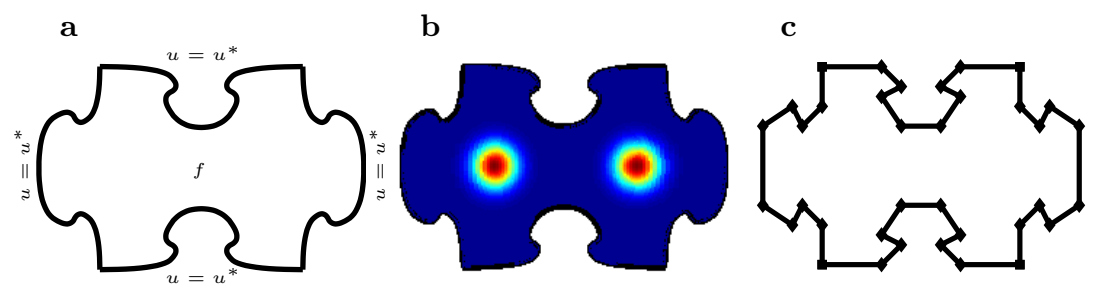

Fig. 10. Jigsaw puzzle. a: Domain and boundary conditions. b: Analytical solution of the boundary value problem. c: Boundary control points.

We solve the parametrization problem using all six nonlinear methods by optimizing the position of the 64 interior control points, giving us a total of 128 

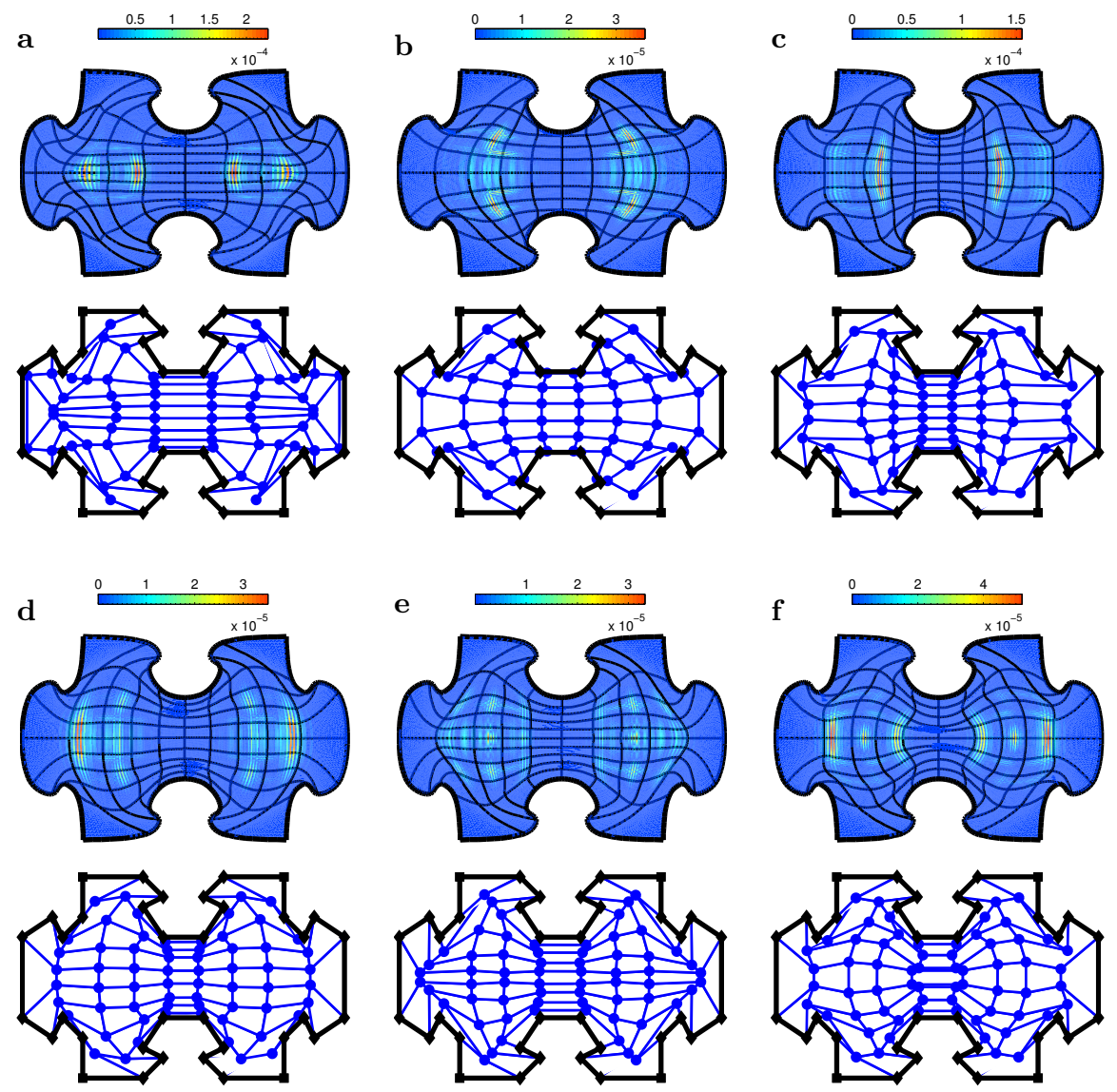

Fig. 11. Jigsaw puzzle piece: Isoparametric lines and numerical error (top) and control net (bottom) for the parametrization based on area-orthogonality (a), Liao (b), Winslow (c), strong residual (d), weak residual (e), and enrichment error (f).

design variables. In this example, we initialize the geometric methods from the spring model in Section 3.1, and the analysis-oriented methods from the Winslow method. The results are shown in Fig. 11, depicting the optimal control net, the corresponding parametrization, and the numerical error. We note firstly that all the optimized control net and their corresponding parametrizations show a high degree of symmetry, as one would expect from the underlying BVP. The parametrizations vary markedly between the methods, and so does the error size and distribution. To examine the numerical error more closely, we compare again the methods in terms of the $L_{2}$-norm of the error when the analysis is refined. This is shown in Fig. 12, displaying the global numerical error $\epsilon$ as a function of the number of degrees-of-freedom for the analysis for each of the six methods. We note that for sufficiently fine discretizations, the global error convergence order is the same for all methods. The superconvergence of the weak residual method 


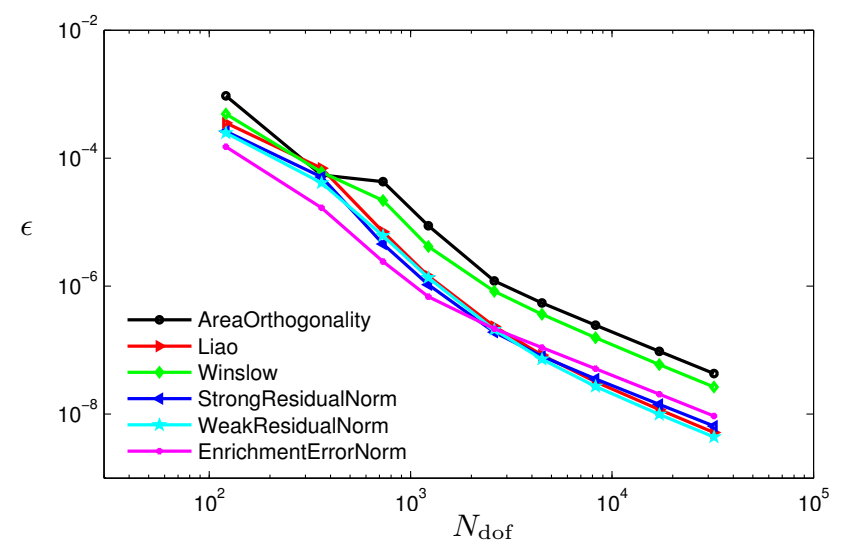

Fig. 12. Jigsaw puzzle: error as a function of number of degrees-of-freedom for the different parametrization methods.

observed in the previous example in Fig. 8 is no longer seen. The difference in the global error varies by approximately one order of magnitude between the methods. Again, the weak residual method yields the lowest error, while the area-orthogonality gives the highest.

In terms of computational expenses, the geometry-oriented methods converged again significantly faster than the analysis-oriented methods. And as each geometric iteration is significantly cheaper than a corresponding analysisoriented iteration, the computational time is orders of magnitude smaller for the geometric methods than the analysis-oriented ones.

\section{Discussion}

The solution to the parametrization problem is particularly important in the context of shape optimization, where a parametrization needs to be recomputed repeatedly as the shape of the physical domain is updated by the shape optimization algorithm, cf. [31]. In addition, most realistic industrial problems can only be realized based on multiple patches, and the problems are most often three-dimensional and not planar. In the present section, we further discuss these challenges.

\subsection{Shape Optimization}

The authors are especially interested in using IGA for shape optimization, which imposes further requirements on the parametrization method. In addition to producing a valid parametrization of high quality, they have to be computationally inexpensive and robust. Last but not the least, they should produce parametrizations, which depend in a differentiable way on the parameters, determining the shape of the domain. For this purpose, the non-linear reparametrization 
methods are often too expensive and too slow in practice. Furthermore, should the numerical algorithm for solving the optimization problems (22) or (31) stop without producing a sufficiently precise stationary point or "jump" from one locally stationary solution to another, the differentiable dependence of the parametrization on the shape parameters might be lost. In order to overcome these problems we have successfully utilized the following approach:

1. First, we find a high quality reference parametrization, employing a possibly expensive non-linear method.

2. During shape optimization iterations, we use a computationally inexpensive linear method and add the validity condition $d_{i, j} \geq \delta Z$, cf. Theorem 1 as constraints to the shape optimization problem. Again, the number $\delta \in[0,1]$ is an algorithmic parameter and the number $Z$ is the result of the optimization $(20)$.

3. If any of the validity constraints in Step 2 is active when the optimization stops, we improve the parametrization by going to Step 1 and restart the optimization.

In the papers $[22,23,26]$ this method has been successfully applied to 2D shape optimization problems. The Winslow functional is minimized in Step 1 and the linearized Winslow functional is used in Step 2, except for [22] where quasi conformal deformation was used.

\subsection{Multiple Patches}

So far we have only considered a single patch, but extending the non-linear methods and their linearizations to several patches is straightforward. We simply let the control points for the inner boundary be variables in the optimization formulations such as (22) and (31). It is interesting to observe how the Winslow functional distributes the angles between patches meeting a common corner, cf. Fig. 13.
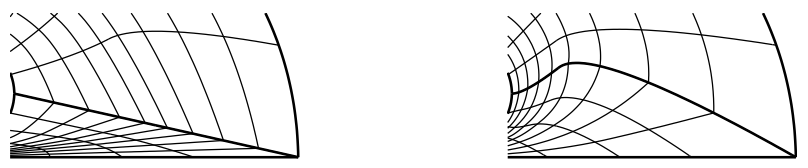

Fig. 13. An inner boundary of a multi-patch configuration. To the left the initial parametrization, to the right the parametrization obtained by minimizing the Winslow functional.

\subsection{Higher Dimensions}

Due to the Radó-Kneser-Choquet theorem the method of minimizing the Winslow functional has a sound mathematical underpinning in dimension two. Unfortunately, there is no version of this theorem in higher dimensions, and there is 
no unique way to generalize the Winslow functional to higher dimensions either. The analysis-oriented methods, on the other hand, generalize verbatim to higher dimensions, as does the Liao Functional.

\section{Conclusion and Outlook}

The construction of geometry parametrizations in isogeometric analysis is of vital importance for obtaining reliable and accurate numerical results. In applications of isogeometric analysis to shape optimization, the requirements to computational algorithms for constructing geometry parametrizations increase further, owing to the repeated updates to the geometry made by the shape optimization process. In the present work, we have proposed several methods, both linear and non-linear, for constructing a parametrization, which meet these requirements.

The linear methods are computationally inexpensive, but do not guarantee that the resulting parametrization is injective. We have outlined the spring model, the mean values coordinates, and the quasi conformal deformation methods. Some of these can be used as an initial guess for other methods, and some work well in the vicinity of a known valid parametrization. The injectivity of the parametrization can be guaranteed by controlling the determinant of the Jacobian, which in turn can be controlled by its coefficients in a B-spline expansion.

Two classes of non-linear parametrization methods have been considered, which are based on maximizing a quantitative measure of the quality of the parametrization. One class is based on the geometric quality measures, and uses some of the methods known from mesh generation. Specifically, we have investigated the area-orthogonality, the Liao, and the Winslow functionals. The other class of quality measures is analysis-oriented, and rely on error estimates. Among many estimators available for adaptive meshing, we have tested three, namely the strong residual, the weak residual, and the enrichment error norm. The non-linear methods require more computational effort than the linear ones, in particular the analysis-oriented methods. At the same time they produce valid parametrizations, typically of higher quality.

We ensure the validity of the parametrization by adding the positivity of the determinant of the Jacobian as constraints to the optimization-based parametrization methods. In our computational experience, these constraints are not active at the end of the optimization, i.e., the functional we minimize has a local minimum in the set of valid parametrizations. This is guaranteed in the case of the Winslow functional which has a unique minimum. To safeguard from numerical errors, we keep the positivity of the determinant of the Jacobian as constraints even in this case.

The analysis-oriented methods strive to make the numerical solution of the $\mathrm{PDE}$ at hand as accurate as possible with respect to a given error estimator. For the few examples of elliptic boundary value problems we have considered, they seem to work well. However, a word of caution is required. Conceivably, instead of making the approximation error smaller we may expose flaws in the error 
estimator and end up with a useless parametrization after all, which nevertheless results in a small estimated error.

We are particularly interested in using isogeometric analysis for shape optimization, and that puts conflicting demands on the parametrization algorithm. It has to be fast, differentiable, robust, and reliable. We have solved the problem by using a cheap and fast linear method most of the time, and only use an expensive non-linear method when it is required. We have considered a range of 2D shape optimization problems and we have successfully used the Winslow functional as the non-linear method and the linearized Winslow functional for the linear method

The future work in the field of parametrizations in isogeometric analysis has both practical/experimental and theoretical aspects. First of all, more tests of the proposed optimization methods are needed on more geometries and other equations, including non-elliptic problems, both in 2D and 3D.

For example, isogeometric analysis is known to perform very well for numerically approximating the eigenvalues, providing the small error even for the optical/high frequency part of the spectrum, apart from a few highest frequency modes [3]. In a simple 1D example with a known spectrum, the error can be made small for all eigenvalues by adjusting the parametrization of the geometry. As eigenvalue approximation errors have far reaching implications for the numerical accuracy of other problems with the same operator, it would be very interesting to know whether such parametrization adjustments generalize to problems in higher dimensions and can be achieved without knowing the exact spectrum.

Another fundamental issue directly related to geometry parametrization is that of generating the patch layout in case several patches are needed. This can be done "by hand," but automated methods are of course highly desirable.

It would be very interesting to characterize the minima of the analysisoriented parametrization methods. For example, for which BVPs/error estimators can we guarantee the validity of the resulting parametrization without explicitly enforcing it?

We believe that no universal linear method for generating a geometry parametrization exists. We formulate it as a conjecture, and the proof of this fact would of course be very interesting:

Conjecture 1. Let $F: C^{1}\left(\partial I^{2}, \mathbb{R}^{2}\right) \rightarrow C^{1}\left(I^{2}, \mathbb{R}^{2}\right)$ be an affine map such that $\left.F(\mathbf{Y})\right|_{\partial I^{2}}=\mathbf{Y}$ for all $\mathbf{Y} \in C^{1}\left(\partial I^{2}, \mathbb{R}^{2}\right)$. Then there is a regular map $\mathbf{Y} \in$ $C^{1}\left(\partial I^{2}, \mathbb{R}^{2}\right)$ with a positive Jacobian determinant in the corners such that $F(\mathbf{Y})$ has a negative Jacobian determinant at least at one point.

Another conjecture is related to minimizing the Winslow functional over the finite-dimensional spaces of splines:

Conjecture 2. Let $\mathbf{Y} \in C^{1}\left(\partial I^{2}, \partial \Omega\right)$ be a valid spline parametrization of the boundary of a domain $\Omega \in \mathbb{R}^{2}$ with a positive Jacobian determinant in the corners. Then there exists a finite-dimensional spline space $\mathcal{S} \subset C^{1}\left(\partial I^{2}, \mathbb{R}^{2}\right)$ and a minimizer $\mathbf{X} \in \mathcal{S}$ of the Winslow functional, such that $\mathbf{X}$ is a valid parametrization of $\Omega$ with $\left.\mathbf{X}\right|_{\partial I^{2}}=\mathbf{Y}$. 


\section{Acknowledgement}

The authors would like to thank Thomas A. Hogan, The Boeing Company, USA, for valuable discussions and for suggesting the enrichment error norm as a quality measure.

\section{References}

1. Choquet, G.: Sur un type de transformation analytique généralisant la représentation conforme et définé au moyen de fonctions harmoniques, Bull. Sci. Math. 69, 156-165 (1945)

2. Cohen, E., Martin, T., Kirby, R.M., Lyche, T., Riesenfeld, R.F.: Analysis-aware modeling: understanding quality considerations in modeling for isogeometric analysis. Comput. Meth. Appl. Mech. Engrg. 199, 334-356 (2010)

3. Cottrell, J.A., Reali, A., Bazilevs, Y., Hughes, T.J.R.: Isogeometric analysis of structural vibrations, Comput. Meth. Appl. Mech. Engrg. 195, 5257-5296 (2006)

4. Cottrell, J.A., Hughes, T.J.R., Bazilevs, Y.: Isogeometric Analysis: Toward Integration of CAD and FEA. John Wiley \& Sons, Chichester (2009)

5. Duren, P., Hengartner, W.: Harmonic Mappings of Multiply Connected Domains, Pac. J. Math. 180, 201-220 (1997)

6. Farin, G., Hansford, D.: Discrete Coons Patches, Comput. Aided Geom. Des. 16, 691-700, (1999)

7. Farrashkhalvat, M., Miles, J.P.: Basic Structured Grid Generation: With an introduction to unstructured grid generation. Butterworth-Heinemann, Burlington (2003)

8. Floater, M.S.: Mean Value Coordinates, Comput. Aided Geom. Des. 20, 19-27 (2003)

9. Floater, M.S., Hormann, K.: Parameterization of Triangulations and Unorganized Points. In Iske, A., Quak, E., Floater, M.S., (eds.) Tutorials on Multiresolution in Geometric Modelling, pp. 287-315. Springer-Verlag, Heidelberg (2002)

10. Floater, M.S., Hormann, K.: Surface Parameterization: a Tutorial and Survey. In Dodgson, N.A., Floater, M.S., Sabin, M.A. (eds.), Advances in Multiresolution for Geometric Modelling, pp. 157-186. Springer-Verlag, Heidelberg (2005)

11. Gravesen, J., Evgrafov, A., Gersborg, A.R., Nguyen, D.M., Nielsen, P.N.: Isogeometric Analysis and Shape Optimisation. In Eriksson, A., Tibert, G. (eds.) Proc. of the 23rd Nordic Seminar on Computational Mechanics, pp. 14-17 (2010)

12. Hormann, k., Greiner, G.: MIPS: An efficient global parametrization method. In P.J. Laurent, P. Sablonnire, and L. L. Schumaker, editors, Curve and Surface Design: Saint-Malo 1999, Innovations in Applied Mathematics, pages 153-162. Vanderbilt University Press, Nashville, TN (2000)

13. Hormann, K., Floater, M.S.: Mean Value Coordinates for Arbitrary Planar Polygons, ACM Transactions on Graphics 25, 1424-1441 (2006)

14. Hughes, T.J.R., Cottrell, J.A., Bazilevs, Y.: Isogeometric Analysis: CAD, Finite Elements, NURBS, Exact Geometry and Mesh Refinement, Comput. Meth. Appl. Mech. Engrg. 194, 4135-4195 (2005)

15. Khattri, S.K.: Grid Generation and Adaptation by Functionals. Comput. Appl. Math. 26, 235-249 (2007)

16. Kneser, H.: Lösung der Aufgabe 41, Jahresber. Deutsch. Math.-Verein. 35, 123-124 (1926) 
17. Knupp, P.M.: Algebraic Mesh Quality Metrics. SIAM J. Sci. Comput. 23, 193-218 (2001)

18. Liao, G.: Variational Approach to Grid Generation. Num. Meth. Part. Diff. Eq. 8, 143-147 (1992)

19. MATLAB. Version 7.14.0.739 (R2012a) The MathWorks Inc. Natick, Massachusetts (2012)

20. Martin, T., Cohen, E., Kirby, R.M.: Volumetric Parameterization and Trivariate B-Spline Fitting Using Harmonic Functions. Comput. Aided Geom. Des. 26, 648 - 664 (2009)

21. Nguyen, D.M., Nielsen, P.N., Evgrafov, A., Gersborg, A.R., Gravesen, J.: Parametrisation in Iso Geometric Analysis: A first report, DTU (2009) http://orbit.dtu.dk/services/downloadRegister/4040813/first-report.pdf

22. Nguyen, D.M., Evgrafov, A., Gersborg, A.R., Gravesen, J.: Isogeometric Shape Optimization of Vibrating Membranes, Comput. Meth. Appl. Mech. Engrg. 200, 1343-1353 (2011)

23. Nguyen, D.M., Evgrafov, A., Gravesen, J.: Isogeometric Shape Optimization for Electromagnetic scattering problems . Prog. in Electromagn. Res. B 45, 117-146 (2012)

24. Nguyen, T., Jüttler, B.: Parameterization of Contractible Domains Using Sequences of Harmonic Maps. In Boissonnat, J.-D., Chenin, P., Cohen, A., Gout, C., Lyche, T., Mazure, M.-L., Schumaker, L. (eds.) Curves and Surfaces. LNCS vol. 6920, pp. 501-514. Springer, Heidelberg (2012)

25. Nguyen, T., Mourain, B., Galigo, A., Xu, G.: A Construction of Injective Parameterizations of Domains for Isogeometric Applications. In Proc. of the 2011 International Workshop on Symbolic-Numeric Computation, 149-150. ACM, New York (2012)

26. Nørtoft, P., Gravesen, J.: Isogeometric Shape Optimization in Fluid Mechanics, Struct. Multidiscip. Opt., doi: 10.1007/s00158-013-0931-8.

27. Octave community: GNU/Octave. www.gnu.org/software/octave (2012)

28. Radó, T.: Aufgabe 41. Jahresber. Deutsch. Math.-Verein. 35, 49 (1926)

29. Sheffer, A., Praun, E., Rose, K.: Mesh Parameterization Methods and their Applications, Foundations and Trends in Computer Graphics and Vision 2, 105-171 (2006)

30. Wächter, A., Biegler, L.T.: On the Implementation of a Primal-Dual Interior Point Filter Line Search Algorithm for Large-Scale Nonlinear Programming. Math. Program. 106, 25-57 (2006)

31. Wall, W.A., Frenzel, M.A., Cyron, C.: Isogeometric Structural Shape Optimization, Comput. Meth. Appl. Mech. Engrg. 197, 2976-2988 (2008)

32. Weber, O., Ben-Chen, M., Gotsman, C., Hormann, K.: A complex view of barycentric mappings. Computer Graphics Forum 30, 1533-1542, Proc. of SGP (2011)

33. Winslow, A.: Numerical Solution of the Quasilinear Poisson Equation in a Nonuniform Triangle Mesh, J. Comput. Phys. 2, 149-172 (1967)

34. Xu, G., Mourrain, B., Duvigneau, R., Galligo, A.: Optimal Analysis-Aware Parametrization of Computational Domain in Isogeometric Analysis. In Mourrain, B., Schaeffer, S., Xu, G. (eds.) Advances in Geometric Modeling and Processing, pp. 236-254. Springer, Heidelberg (2010)

35. Xu, G., Mourrain, B., Duvigneau, R., Galligo, A.: Parameterization of Computational Domain in Isogeometric Analysis: Methods and Comparison. Comput. Meth. Appl. Mech. Engrg. 200, 2021-2031 (2011) 